American Journal of Applied Sciences 8 (3): 217-229, 2011

ISSN 1546-9239

(C) 2011 Science Publications

\title{
Heat-Modeling of Microwave Assisted Epoxidation of Palm Acid Oil
}

\author{
N. Saifuddin, L. Wei Zhan and K. Xin Ning \\ Department of Engineering Sciences, College of Engineering, \\ University Tenaga Nasional, Jalan Ikram-Uniten, 43000 Kajang, Selangor, Malaysia
}

\begin{abstract}
Problem statement: While microwave chemistry could well be the most convenient, rapid and energy-saving way to initiate a chemical reaction, the issues of thermal versus microwave specific effect remain unaddressed. Approach: A three-dimensional Finite Element Model (FEM) was developed to predict temperature profile during the enzymatic epoxidation of palm acid oil using microwave heating. Three-dimensional partial differential equations are formulated for the electromagnetic field and heat and mass transfer processes. The temperature profile of the FEM generated from the commercial software (ANSYS Emag) was verified using experimental data from microwave oven heated samples containing palm acid oil and reactants in cylindrical bottles and has compared favorably with real time data. An Infra Red thermometer was used to measure temperature of the reactants during the real time experiment to ensure less contact with the reaction vessel. Results: Apart from ascertaining the temperature profile of the epoxidation process in the cylindrical bottle, the heat-modeling of this reaction can predict the Energy required during the reaction and provide insights to non-thermal microwave effects. It has shown the possibility of Microwave Non-Thermal Effects and Microwave Selectivity in Substrate Specificity. The energy used by the microwave irradiated reaction is by far much lesser than the energy used in conventional heating. This study has also improved the epoxidation process of Palm Acid Oil by incorporating enzyme as a catalyst and using microwave heating. Epoxidation can be done rapidly and at a lower level of activation energy of $27.767 \mathrm{~kJ} \mathrm{~mol}^{-1}$. It is a green process with increased efficiency as it reduced a typically 5-hour reaction to less than an hour. Conclusion: In this study, a quantitative view of a microwave assisted process of enzymatic epoxidation has been established. We have successfully developed and experimentally validated a 3D electromagnetic heat transfer model. This model can thus be used to predict the energy of the epoxidation and other future microwave reactions. the study also have shown the existence of microwave non-thermal effects and microwave selectivity in substrate specificity.
\end{abstract}

Key words: Microwave chemistry, chemo-enzymatic, non-thermal effects, Palm Acid Oil (PAO), Finite Element Model (FEM), electromagnetic calculations, heat-modeling, energy-saving, activation energy

\section{INTRODUCTION}

Microwave chemistry has been gaining attention in recent years as it provides rapid reaction rates and higher yield of pure products. The selective absorption of microwave energy by polar molecules or polar transition state intermediates formed during the course of the reaction has shown significant increase in reaction rates (Saifuddin and Fazlili, 2009; Caddick and Fitzmaurice, 2009; Jumbe and Nandini, 2009). Apart from organic synthesis, MW heating has been applied in fields such as organometallic synthesis, homogeneous and heterogeneous catalysis, phase transfer catalysis, organocatalysis and biocatalysis (Jumbe and Nandini, 2009).

Conventional heating such as conduction, convection and heat radiation has a heat source on the outside and relies on the heat transfer process to eventually reach the core. Microwave dielectric heating, however, provides the following advantages for chemical synthesis (Li and Yang, 2008):

- The introduction of microwave energy into a chemical reaction can lead to much higher heating rates than those which are achieved conventionally

- Microwave energy is introduced into the chemical reactor remotely without direct contact between the energy source and the reacting chemicals

- It is volumetric and instantaneous (or rapid) heating with no wall or heat diffusion effect It can achieve selective heating because chemicals and the containment materials for chemical reactions do not interact equally with microwaves. Specifically the containment materials for a chemical reaction may be chosen in such a way that the microwave energy passes

Corresponding Author: N. Saifuddin, Department of Engineering Sciences, College of Engineering,

University Tenaga Nasional, Jalan IKRAM-UNITEN, 43000 Kajang, Selangor, Malaysia 
through the walls of the vessel and heats only the reactants. For example, in the case sintering of metal powder, the very high temperatures which result when metal powders are exposed to microwave fields have been used to create hot spots' which accelerate the reactions of the metals with gases, other inorganic solids and organic substrates.

- These selective interactions mean that microwave dielectric heating is an ideal method for accelerating chemical reactions under increased pressure conditions

The selective absorption of microwave energy by polar molecules or polar transition state intermediates formed during the course of the reaction has shown significant increase in reaction rates (Caddick and Fitzmaurice, 2009; Horikoshi and Serpone, 2009; MacKenzie et al., 2009). Numerous experiments on reaction rate enhancement in microwave field, especially with solid-state reactions have been observed. Although many point to the fact that this occurrence cannot be interpreted by thermal effects of microwave solely, it had been pointed out that the energy of microwave photon $\left(\approx 1 \mathrm{~J} \mathrm{~mol}^{-1}\right)$ is far too small to break typical chemical bands (usually $>300$ $\mathrm{J} \mathrm{mol}^{-1}$, even the energy of hydrogen bond is several tens of Joules per mole) (Li and Yang, 2008). This leads to defining the concept of non-thermal effects, also called "specific microwave effect". According to Pillai et al., (2002) olefin epoxidation occurs readily upon MW exposure due to the polar nature of the reaction intermediates that couple efficiently with microwaves and hence the dramatic rate acceleration and not due to any specific microwave effect. Kappe (2004) also, concluded that all the speculations on special and non-thermal effects in microwave heating has no basis when taking into account the increased temperatures caused by superheating or concentration effect.

However, there had also been clear reports that microwave reaction improves completion within minutes of what usually takes hours. Huang et al. (2005) reported that there is microwave effect on substrate specificity of alcohol, increasing the reaction rate rapidly (Huang et al., 2005). There are also reports that microwave heating could result in different membrane morphology, orientation, composition for the production of zeolite membranes. And therefore have different permeation characteristics as compared with conventional heating synthesized ones. It was concluded that the function of microwave irradiation during zeolite membrane formation is mainly derived from its thermal effect alongside specific microwave effects, such as "activewater", "selective activation".
The microwave method of chemical reactions is relatively new as opposed to conventional thermal heating. Due to the fact that microwave heating is a common domestic method used for cooking, the vast majority of studies researching the effects of microwave-irradiation on oil structure and properties have focused on the changes in the lipids of the food itself. Many other studies have exposed seed crops to microwave irradiation prior to any extraction processes, but few studies have focused on the changes in structure and properties of microwave irradiated purified oil. In these studies, pressure and temperature measurements inside the microwave cavity, pose a certain challenge as limited devices can be utilized without interfering or damaging the magnetron or the device itself. More often than not, the control and setting of reaction parameters is limited to the energy input and the irradiation time. Accuracy of the measurements is also in question as the most common method of using radiometry often provides different temperature feedbacks at different depths. While microwave chemistry could well be the most convenient, rapid and energy-saving way to initiate a chemical reaction, these limitations remain unaddressed and until more detailed studies are made and comprehensive findings are more widely found, a comparison with classical reaction conditions is difficult and probably the reason to why speculations about non-thermal (or microwave) effects are a rife. With this in mind, there is need to develop a 3D Finite Element model to better study the energy that the electromagnetic wave (microwave) imparts on the reactants and its effect (Thomas and Dozier, 2010).
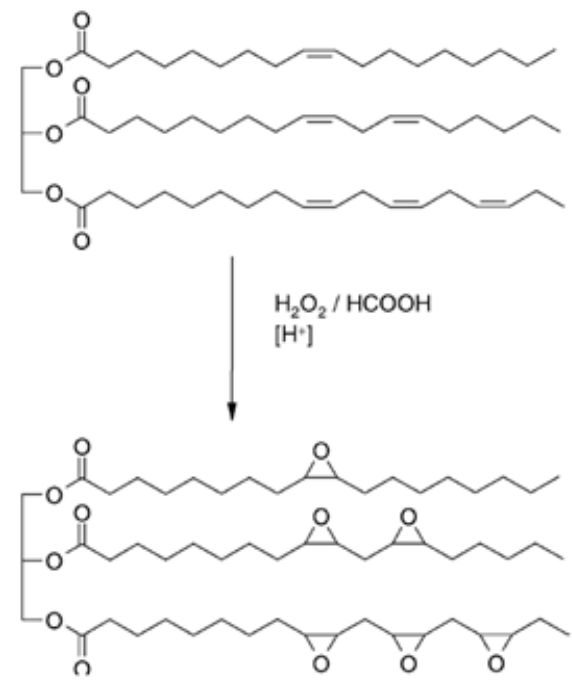

Fig. 1: Epoxidation reaction of vegetable oils 


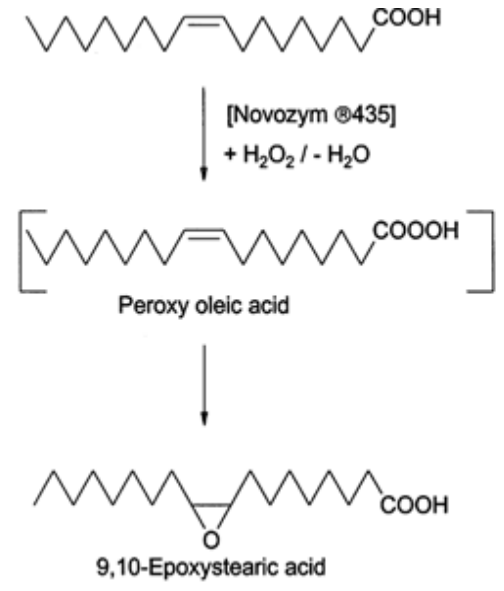

Fig. 2: Chemo-enzymatic 'self'-epoxidation of unsaturated fatty acids; conversion of oleic acid to epoxystearic acid via peroxy oleic acid (Rüsch gen and Warwel, 1999)

Vegetable based oils often exhibit superior attributes and as in the case of lubricants, vegetable oil based products have better anti-wear properties, higher lubricity, higher viscosity index, non-toxic and ultimately, causes no pollution. However, they also have drawbacks that must be overcome, including poor low-temperature properties such as opacity, precipitation, poor flowability and/or solidification at relatively moderate temperature, their susceptibility to oxidative degradation and their tendency to undergo hydrolysis in acid media (Hwang et al., 2003). As such, chemical modifications to the vegetable oil must be done to improve usability.

Epoxidized oils are currently produced on an industrial scale by the Prileshajev epoxidation reaction in which a peracid is used for oxygen transfer to the double bonds (Fig 1).

A short-chain peroxy acid, usually peracetic acid is prepared from hydrogen peroxide and acetic acid either in a separate step or in situ. Large scale epoxidation usually avoid the handling of peroxy acids by using the in situ method but this requires strong mineral acid to act as a catalyst in the forming of the peroxy acid. The presence of strong mineral acid causes side reactions and thus the selectivity rarely exceeds $80 \%$. Enzymes are capable of lowering activation energy by temporarily combining with the chemicals involved in the reaction, acting as a reaction site. The reaction is summarized in Fig 2 (Rüsch gen and Warwel, 1999). Tornvall et al. (2007) had showed that Novozym 435 is capable of catalyzing the conversion of fatty acids with hydrogen peroxide to peroxy fatty acids. In this method of 'self-epoxidation' of unsaturated fatty acids, up to $91 \%$ yield and more than $98 \%$ selectivity has been reported. In the chemo-enzymatic epozidation process, only a minimum amount of enzyme was necessary to show the catalytic effect and up to six rounds of usage can be achieved with reported optimum enzyme activity (Orellana-Coca $e t$ al., 2005).

The objective of this study is : To perform an FEA on the heat model for the microwave irradiated enzymatic epoxidation process and to investigate non-thermal microwave effects on epoxidation process.

\section{MATERIALS AND METHODS}

Materials: Palm Acid Oil (kindly supplied by Unitata Refinery, Ipoh, Malaysia) was used as the starting material for chemical modification. Formic acid $(99.81 \%)$, Ethyl Acetate, Novozym 435 and Hydrogen Peroxide (30\%) were ACS grade and purchased from Sigma-Aldrich and J.T. Baker. Water was Ultra-filtered and deionized using ELGA LabWater (UK) water purification system.

Preparation of reactants before incorporating microwave irradiation: Palm Acid Oil (20g) was first heated to remove moisture and left to cool until $40^{\circ} \mathrm{C}$. Next, $45 \mathrm{ml}$ of Ethyl Acetate and $0.3 \mathrm{~g}$ of Formic Acid were added with $50 \mathrm{mg}$ Novozym 435, under moderate stirring. The mixture was so kept for $15 \mathrm{~min}$. After which $3.9 \mathrm{ml}$ of Hydrogen Peroxide (in excess, with molar ratio of $\mathrm{C}=\mathrm{C}$ to $\mathrm{H}_{2} \mathrm{O}_{2}$ of $1: 20$ ) was added dropwise for 15 minutes. Although the concentration of the peroxy fatty acid ester formed is detrimental to enzyme activity, the rapid process ensures that the enzyme action has not declined while epoxidation is taking place during the onehour duration.

Microwave irradiation: Epoxidation was carried out in closed borosilicate (nearly microwave transparent) reaction vessels ( $250 \mathrm{~mL}$ capacity) in a microwave oven $(2.45 \mathrm{GHz})$ equipped with magnetic stirring and heated according to the temperature settings shown in Table 1. The MW power input was optimized for the different solvents and substrates in order to reach the required temperatures in 30-40 s, without temperature overshooting. The effects of water present in the solvents and reagents (used as received) are neglected for the sake of simplicity. Zero time was taken as the desired operation mode was initiated. However the temperature has to be checked from time to time to ensure it has not exceeded its range. Hence, an Infrared thermometer was used to accurately check the temperature of the reaction mixture. Power settings were based on adjustments according to temperature of reaction needed. The mixture was reacted for five 6minute cycles, making a total of $30 \mathrm{~min}$. 
Table 1: Temperature of reaction vessel and related power settings

\begin{tabular}{|c|c|c|}
\hline $\begin{array}{l}\text { Average } \\
\text { temperature }\left({ }^{\circ} \mathrm{C}\right)\end{array}$ & Deviation $( \pm)$ & Power settings \\
\hline $45^{\circ} \mathrm{C}$ & $3^{\circ} \mathrm{C}$ & $1 \min 270 \mathrm{w}, 5 \mathrm{~min} 90 \mathrm{w}$ \\
\hline $55^{\circ} \mathrm{C}$ & $3^{\circ} \mathrm{C}$ & $2 \min 270 w, 4 \min 90 w$ \\
\hline $65^{\circ} \mathrm{C}$ & $3^{\circ} \mathrm{C}$ & $2: 30 \min 270 w, 3: 30 \min 90 \mathrm{w}$ \\
\hline $75^{\circ} \mathrm{C}$ & $3^{\circ} \mathrm{C}$ & $3 \min 270 w, 3 \min 90 w$ \\
\hline $85^{\circ} \mathrm{C}$ & $3^{\circ} \mathrm{C}$ & $3: 30 \min 270 w, 2: 30 \min 90 w$ \\
\hline
\end{tabular}

Table 2: Simulation input parameters

\begin{tabular}{ll}
\hline Parameter & Value \\
\hline Oven interior dimension & $37 \times 22.5 \times 33.5 \mathrm{~cm}$ \\
Reaction vessel dimension & $\begin{array}{l}\mathrm{H}: 6 \mathrm{~cm} \mathrm{rad:} 4 \mathrm{~cm} \\
\text { (Assume cylindrical) }\end{array}$ \\
Placement & Center \\
Heating power & Varied (Table\#) \\
Thermal and electromagneti & \\
c properties & \\
Thermal conductivity & $0.1546 \mathrm{~W} \mathrm{~m}^{-1{ }^{\circ} \mathrm{C}}$ \\
Density & $867.307 \mathrm{~kg} \mathrm{~m}^{-3}$ \\
Specific heat & $2492.173 \mathrm{~J} \mathrm{~kg}^{-1{ }^{\circ} \mathrm{C}}$ \\
Dielectric constant & 5.1 \\
Dielectric loss & 1.6 \\
Heat transfer coefficient & 691 \\
Microwave frequency & $2.45 \mathrm{GHz}$ \\
Waveguide dimensions & $10 \times 12 \mathrm{~cm}$ \\
Microwave excitation & 20 \\
\hline
\end{tabular}

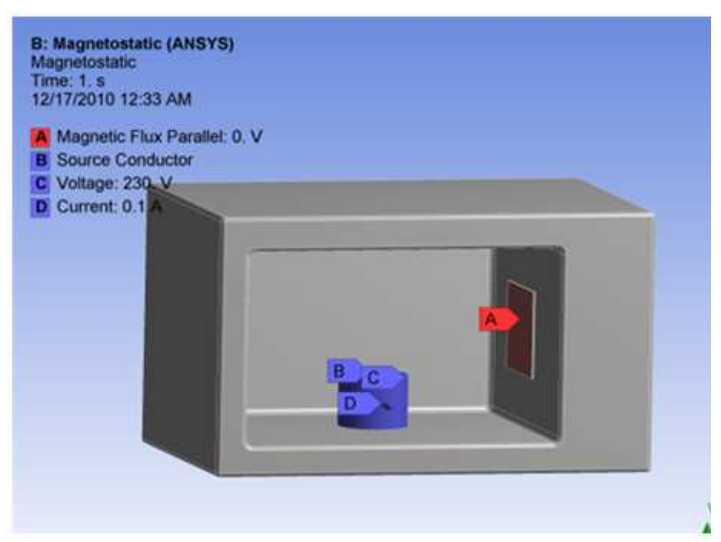

(a)

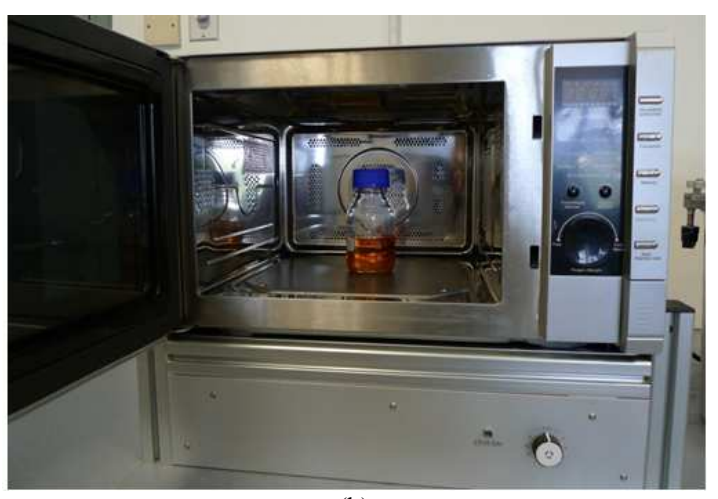

(b)

Fig. 3: Microwave set up diagram
The MW irradiation was interrupted for sampling, which took approx. $45 \mathrm{~s}$ from the instant the MW irradiation was interrupted to the instant the reaction temperature set point was reached causing a decrease in temperature of less than $1{ }^{\circ} \mathrm{C}$. The apparent activation energies and pre-exponential factors were estimated applying the method of initial rates and the Arrhenius equation: the volume of the reaction mixture and the catalyst:oxidant:olefin ratio was kept constant and initial rates were calculated.

Finite element heat model: Figure 3 shows the Electromagnetic 3D model before the simulation was run. The waveguide, where the source of electromagnetic wave (microwave) would be emitted, is tagged as " $\mathrm{A}$ ". The reaction mixture was approximated to be a cylinder as the borosilicate bottle containing it is near microwave transparent. As such the cylinder is approximated to have a radius of $40 \mathrm{~mm}$ and a height of $60 \mathrm{~mm}$. As the simulation runs, wave emitted from the waveguide would be absorbed by the reaction mixture with minimal (negligible) leakage. The power absorbed by the mixture would be a function which would then be used to calculate the temperature achieved. The simulation input parameters are as shown in Table 2.

Governing equations: The equations that govern microwave heating of a material are Maxwell's equations. They govern the propagation of the microwave radiation and the forced heat equation, which in turn, governs the absorption and diffusion of heat by the material. If the material is assumed to be homogeneous, isotropic and Ohmic (current $\mathbf{J}$ and the displacement current $\mathrm{D}$ are both proportional to the electric field $\mathrm{E}$ and that the magnetic flux density $\mathrm{B}$ is proportional to the magnetic field strength $\mathrm{H}$ ), then Maxwell's equations of electromagnetism constant permittivity and permeability and with no sources can be written as:

$\nabla \times \overrightarrow{\mathrm{E}}=-j \omega \mu \overrightarrow{\mathrm{H}}$

$\nabla \times \overrightarrow{\mathrm{H}}=-j \omega \varepsilon_{0} \varepsilon \overrightarrow{\mathrm{E}}$

$\nabla \times \overrightarrow{\mathrm{E}}=0$

$\nabla \times \overrightarrow{\mathrm{H}}=0$

In general, all the material properties are temperature-dependent. For a plane wave propagating in the positive $\mathrm{x}$-direction, it can be assumed that the electric field is in the $\mathrm{y}$-direction and the magnetic field is in the $\mathrm{z}$-direction. Here, $\overrightarrow{\mathrm{E}}$ is the electric field intensity and $\overrightarrow{\mathrm{H}}$ is the magnetic field intensity, both defined as time harmonics Eq. 5 and 6:

$$
\begin{aligned}
& \vec{E}(x, y, z, t)=\overrightarrow{E 0}(x, y, z,) e^{j \omega t} \\
& \vec{H}(x, y, z, t)=\overrightarrow{H_{0}}(x, y, z,) e^{j \omega t}
\end{aligned}
$$




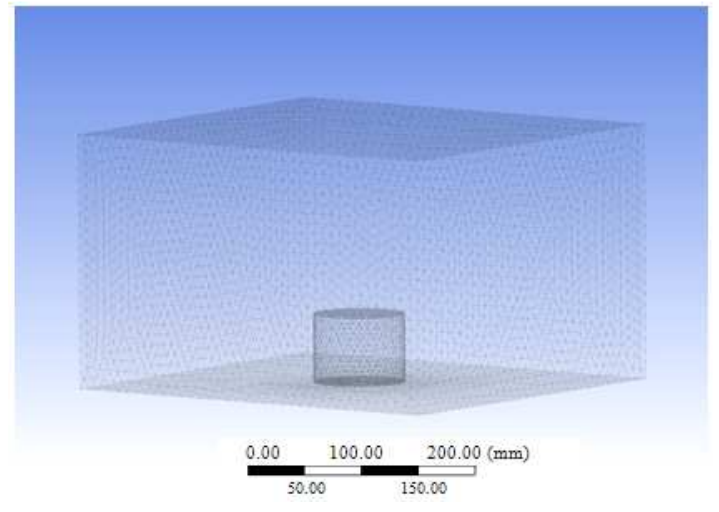

Fig. 4: Mesh of 3D electromagnet model with reaction vessel

The tangential component of electric field is continuous at an interface. In a microwave cavity, boundary conditions need to be specified on the walls of the cavity which are considered to be perfect electrical conductors. Inside a perfect conductor, electric field is zero. The two conditions lead to following boundary conditions on the airwall interface:

$$
\mathrm{E}_{\text {tan gential }}=0
$$

In general, Maxwell's nonlinear equations are intractable and various simplifying assumptions are necessary to obtain analytical mathematical solutions. Eq. 1-2 can be expressed solely in terms of electric field intensity, $\overrightarrow{\mathrm{E}}$, using Eq. 3-4:

$$
\nabla^{2} \overrightarrow{\mathrm{E}}+\omega^{2} \mu \varepsilon_{0} \varepsilon \overrightarrow{\mathrm{E}}=0
$$

The excitation for the microwave oven is through a rectangular waveguide. The shape of the waveguide is designed to transmit maximum possible power from the magnetron to the cavity and the dominant mode of the Transverse Electric (TE) waves is the $\mathrm{TE}_{10}$ mode.

Power density is the power absorbed per unit volume, $P$, by the material. It is related to the electric field by the following equation:

$$
\mathrm{P}(\mathrm{x}, \mathrm{y}, \mathrm{z}, \mathrm{t})=\frac{1}{2} \omega \varepsilon_{0} \varepsilon^{\prime \prime}|\overrightarrow{\mathrm{E}}|
$$

where $\omega$ is the angular frequency and $|\vec{E}|$ is the RMS magnitude of the electric field in the material.

Microwaves heat materials volumetrically and hence, power absorbed by the material due to application of microwaves is added as a source in the heat transfer equation. The transient diffusion equation with the microwave source term is solved to obtain temperatures inside the material:
$\mathrm{Pc}_{\mathrm{p}} \frac{\delta \mathrm{T}}{\delta \mathrm{t}}=\mathrm{k} \nabla^{2} \mathrm{~T}+\mathrm{P}(\mathrm{x}, \mathrm{y}, \mathrm{z}, \mathrm{t})$

Boundary Conditions: Heat loss due to convection and evaporation:

$\mathrm{K} \nabla \mathrm{T} . \mathrm{n}=-\mathrm{h}\left(\mathrm{T}_{\mathrm{a}}-\mathrm{T}_{\mathrm{s}}\right)-\frac{\mathrm{m}_{\mathrm{v}}}{\mathrm{A}} \Delta \mathrm{H}_{\mathrm{v}}$ for $\mathrm{t}>0$

Some amount of air flows over the surface of the food inside the oven due to the presence of a fan. The main function of the fan is to cool the magnetron present in the oven. Heat loss due this airflow is modelled by specifying the convective heat transfer coefficient at the surface of the material (Geedipalli, 2007):

$-\mathrm{k} \nabla \mathrm{T}=\mathrm{h}_{\mathrm{c}}\left(\mathrm{T}-\mathrm{T}_{\mathrm{a}}\right)$

$\mathrm{h}_{\mathrm{c}}=$ Convection heat transfer coefficient over a surface

$\mathrm{T}_{\mathrm{a}}=$ Temperature of air inside the oven

Eq. 8 is solved using the FEM based commercial code ANSYS v12.1 with boundary conditions given by Eq. 7. $\overrightarrow{\mathrm{E}}$ is obtained and $\mathrm{P}(x, t)$ is calculated using Eq. 9. The finite element mesh consisted of tetrahedral second order volume elements with 10 nodes per element and the degree of freedom as the projection of the electric field, $\overrightarrow{\mathrm{E}}$ on edges and faces of the element. The total number of elements used is 401,000 and the number of nodes is 72,500 .

The governing equation for heat transfer Eq.(10) was solved in another FEM based commercial solver, Transient Thermal. The computational domain for the thermal simulations included the reaction vessel only. Hexahedral elements were used for volume meshing for heat transfer analysis and the mesh had 540 elements with 780 nodes. Figure 4 is a mesh of 3D electromagnet model with reaction vessel. The power density term obtained as a function of time from electromagnetic calculations (in ANSYS) was inputted at each node of the mesh for heat transfer simulations as the source term Eq. (9). The transient heat transfer equation was solved with the boundary conditions given by Eq. $11-12$ to obtain the temperatures inside the vessel. The total time of heating was 30 minutes.

In return, the power density term that has been obtained as a function of time from electromagnetic calculations (in ANSYS) and will then be inputted at each node of the mesh for heat transfer simulations as the source term. To validate the computational model, experimentally measured 
values of temperature were compared to the calculated values obtained from the model.

\section{RESULTS AND DISCUSSION}

Palm Acid Oil: Fatty Acids have been reported to increase lubricity of a material. The film-forming properties of these molecules are believed to inhibit metal-to-metal contact and progression of pits and asperities on the metal surface. Strength of the protective fluid film and extent of adsorption on the metal surface dictate the efficiency of a lubricant's performance. In chemical terms, native and synthetic esters exhibit the same structure. Lubricity is greatly increased because the fatty acids tend to migrate from the oil to the surface of the part where vacant sites are available. They adsorb and assemble a protective film till they are removed by subsequent contact with counter-surfaces. The assembly and desorption or wear and reassembly is a dynamic process. Furthermore, some other works report a reduction in friction for the fatty acids with humidity. However, if a group of fatty acids in the adsorbed state is removed by the sliding of the surfaces and the adsorption kinetics does not allow the reassembly to occur before the next contact occurs, the film is not protective and the friction tends to be that corresponding to bare metallic contact (Sahoo and Biswas, 2009).

Bio-based lubricants are derived from vegetable oils, thus there always exists an ethical debate on the idea of using edible oils for non-edible products as palm oil is widely used for cooking in Southeast Asia and the tropical belt of Africa. However, as opposed to using palm oil, the use of palm acid oil, which is a by-product, to produce bio-lubricant is adding value by diversifying its uses. Palm Acid Oil is a byproduct of the refining process of palm oil. It is used for making laundry soaps and for producing calcium soaps for animal feed formulations. The main components of Palm Acid Oil (PAO) are free fatty acids, neutral oil and moisture (Kuntom et al., 1994).

Palm Acid Oil proves to be a suitable candidate for the base material for lubricant as it has very high Free Fatty Acid contents is not edible and is costbeneficial as the current uses are few. The properties of Palm Acid Oil are shown in Table 3. The Table 4 shows the calculated values of Percent Unsaturation, Molecular Weight and Free Fatty Acid content of Palm Acid Oil.

The optimum temperature for enzymatic epoxidation assisted by microwave: The chemoenzymatic epoxidation process is highly dependent on initial reaction rates. However, moderate temperatures were reported to yield minimum epoxide degradation (Honghai et al., 2007). The experimental results of the chemo-enzymatic epoxidation of Palm Acid Oil by performic acid at different temperatures are presented in Fig. 5. As expected, the velocity of the reaction increases with temperature. But even though the peracid is completely consumed in the reactions at 75 and $85^{\circ} \mathrm{C}$, a full yield with respect to the initial double bond concentration is not reached. This observation is explained by the possible decomposition of the peracid. Therefore, in the evaluation of the experimental data this effect is considered. The chemo-enzymatic epoxidation of Palm Acid Oil below $65^{\circ} \mathrm{C}$ with $30 \% \quad \mathrm{H}_{2} \mathrm{O}_{2}$ deactivation of the lipase is not expected because it is within the range of the enzyme's temperature tolerance. The results indicate that Epoxidation of PAO is more complete at higher temperatures with $0.96 \%$ of epoxy content by weight. This value surpasses the predicted value (according to percentages of unsaturated fatty acids and Iodine value of PAO listed in Table 3, full epoxidation is predicted to yield $0.433 \%$ ) more than doubly. As the Hydrogen Peroxide used was in excess to $\mathrm{C}=\mathrm{C}$ content ratio, it is therefore assumed that epoxidation has taken place fully. It is also noticed that after the epoxy content peaks, it tends to go on a decline.

This is the due to the fact that at higher temperatures of reaction, oxirane rings are susceptible to ring-opening. Hence $55^{\circ} \mathrm{C}$ is taken as the optimum temperature of reaction. The higher oxirane content obtained in this study is not unusual as there are reports which indicate heating under microwave irradiation greatly increased acid value, peroxide value, dielectric point of polar compound than with untreated and conventional heating (Albi et al., 1997; Biswas et al., 2007). There is also report that microwave irradiation would reverse the effect, i.e. giving lower iodine and peroxide values (Tan et al., 2001). However, it has been shown that close monitoring of the temperature during microwave irradiation is crucial in obtaining higher values of the parameters mentioned (Biswas et al., 2007). Therefore careful monitoring of the temperature had been carried out to avoid the reverse effects reported by Tan et al. (2001). The increase of epoxy content in this study also indicates that microwave irradiation increases the peroxy acid value thus increasing the eventual oxirane value. Results obtained shows that there are in fact more sites of unsaturation than assumed in Palm Acid Oil as compared with results from conventional heating.. It is proposed that microwave irradiation tends to orientate the hydrocarbon chain in such a way that exposes more of the $\mathrm{C}=\mathrm{C}$ bonds which in turn would increase the iodine and oxirane values. This proves the existence of microwave non-thermal effect in enhancing the epoxidation reaction. 
Am. J. Applied Sci., 8 (3): 217-229, 2011

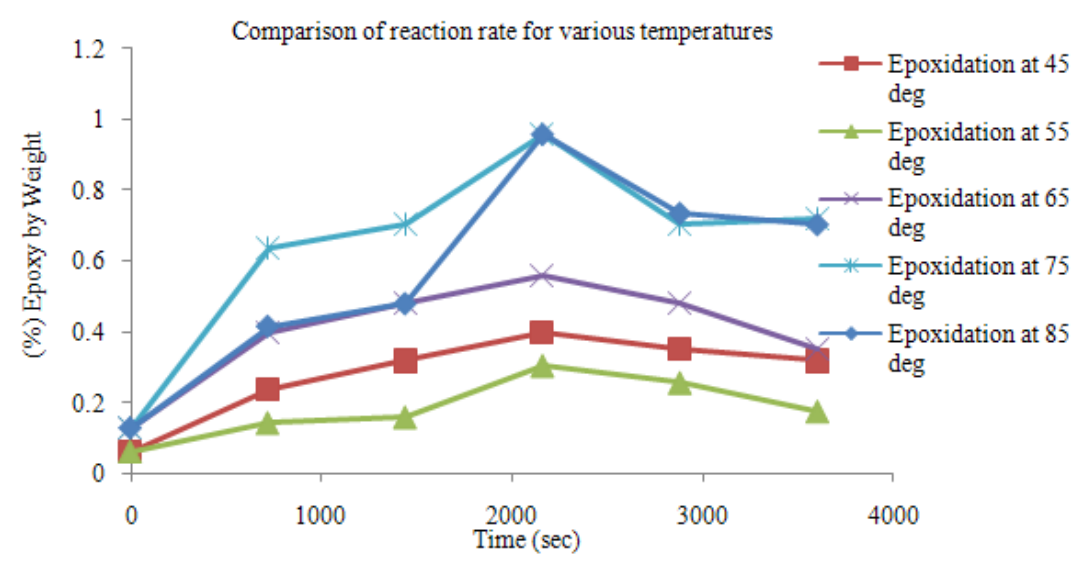

Fig. 5: Oxirane oxygen content at $45,55,65,75$ and $85^{\circ} \mathrm{C}$

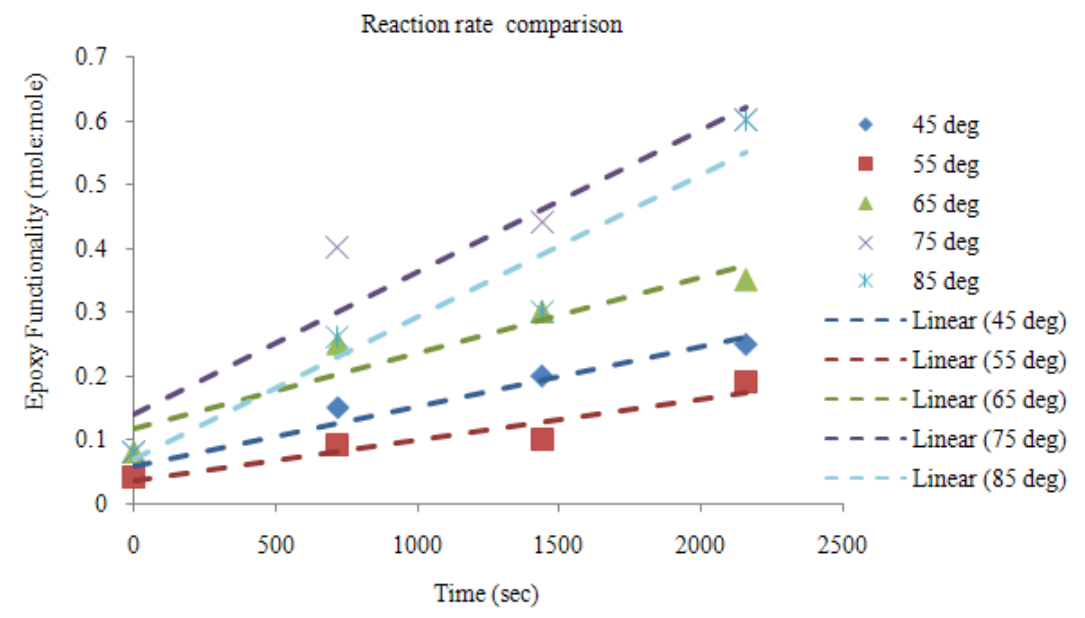

Fig. 6: Reaction rate at various temperatures

Table 3: Properties of palm acid oil oil (Kuntom et al., 1994)

\begin{tabular}{lccr}
\hline & $\begin{array}{c}\text { Mean value of } \\
\text { 27 samples }\end{array}$ & $\begin{array}{c}\text { Standard } \\
\text { deviation }\end{array}$ & Range \\
\hline Moisture content (\%) & 0.98 & 0.53 & 54.08 \\
Free fatty acid (\%) & 62.60 & 11.50 & 18.49 \\
Peroxide value $\left(\mathrm{meq} \mathrm{kg}^{-1}\right)$ & 4.10 & 3.80 & 92.68 \\
Iodine Value & 50.20 & 5.30 & 10.56 \\
Saponification value & 186.00 & 5.60 & 3.01 \\
Unsaponifiable matter & 0.53 & 0.42 & 79.25 \\
\hline
\end{tabular}

Table 4: Percent unsaturation, molecular weight, free fatty acid content of palm acid oil

\begin{tabular}{|c|c|}
\hline Parameter & Value \\
\hline (\%) Neutral oil (after removal of moisture) & $37.4(\%)$ \\
\hline Unsaturation $(\mathrm{C}=\mathrm{C})$ & $0.187 \mathrm{~mol}^{-1}$ \\
\hline Molecular weight of PAO & $468.93 \mathrm{~g} \mathrm{~mol}^{-1}$ \\
\hline
\end{tabular}

The reaction rates shown Fig. 6 at various temperatures strongly suggest that better yield can be attained at higher temperatures. However, due to the presence of enzyme as a catalyst in the reaction, a temperature of more than $80^{\circ} \mathrm{C}$ might hinder its activity. By calculating the epoxy functionality, the reaction rates of each temperature set and the slope of the line for (ln k) vs. (1/T) according to the Arrhenius Equation, the Activation Energy $\left(\mathrm{E}_{\mathrm{a}}\right)$ is found to be $27.767 \mathrm{~kJ} \mathrm{~mol}^{-1}$. This value is much lower than the $\mathrm{E}_{\mathrm{a}}$ needed for the epoxidation of other types of oil, further favoring the use of PAO as the base oil for a chemoenzymatically modified lubricant. The use of Novozym $435^{\circledR}$ as a catalyst reduces the Activation Energy needed for the reaction as well as increases the selectivity, thus producing minimal by-products at such high temperatures. The specific nature of this enzyme increases yield tremendously and will prove promising if it is to be used in a large scale production.

\section{Simulation outcome:}

Power absorbed: The power density term that has been obtained as a function of time from electromagnetic calculations:

$$
\mathrm{P}(\mathrm{x}, \mathrm{y}, \mathrm{z}, \mathrm{t})=\frac{1}{2} \omega \varepsilon_{0} \varepsilon^{\prime \prime}|\overrightarrow{\mathrm{E}}|^{2}
$$




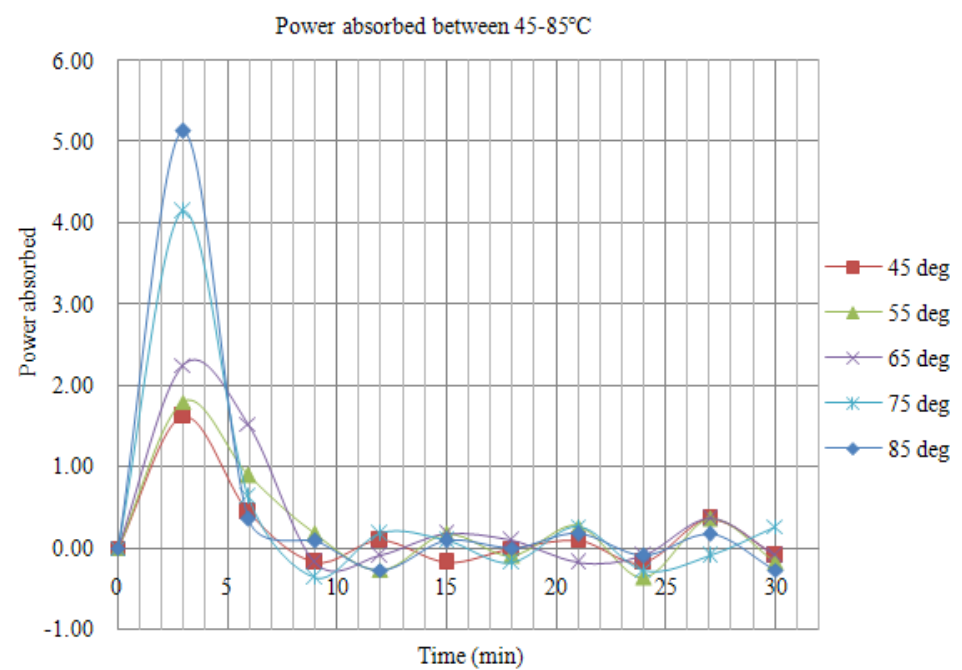

Fig. 7: Power absorbed by reaction at various temperatures

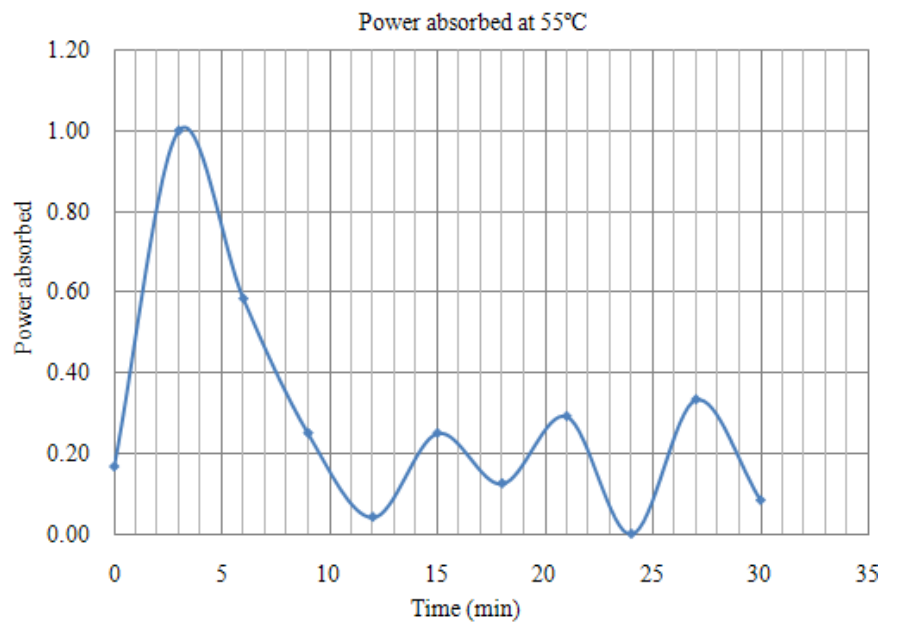

Fig. 8: Normalized power absorbed per unit volume at $55^{\circ} \mathrm{C}$

$\mathrm{P}$ represents the heat generation term. It is this equation that connects the electromagnetic waves with heat transfer phenomenon.

Fig. 7 shows the power density of the chemoenzymatic epoxidation (non-normalized). This comparison shows the level of energy absorbed by the different reactions at different temperatures. The spike in the $3^{\text {rd }}$ minute is indicative of the efficiency of microwaves to heat the material to the temperature required volumetrically. At this point, the temperature of the reaction mixture has reached the indicated temperature.

After the power density term that has been obtained, a set of temperature data can be generated and as seen in Fig. 8 the set of simulated power absorbed data had been translated to the temperature curve and can be compared to the experimental temperature data.
To validate the computational model, experimentally measured values of temperature were compared to the calculated values obtained from the model. Figure 9 presents the measured and calculated temperature histories of the reaction mixture as a whole. The values are found to match at the end of each 30 minute heating interval with some deviation in the experimental and computed values at intermediate times. This may be attributed to the method of how the temperature data was collected. In the absence of a thermocouple to precisely obtain online temperature of the mixture while the experiment was running, the use of an Infra-Red thermometer is more time-consuming and within that timeframe, the heat may have been diffused to the ambient environment causing slight drops in temperatures. 


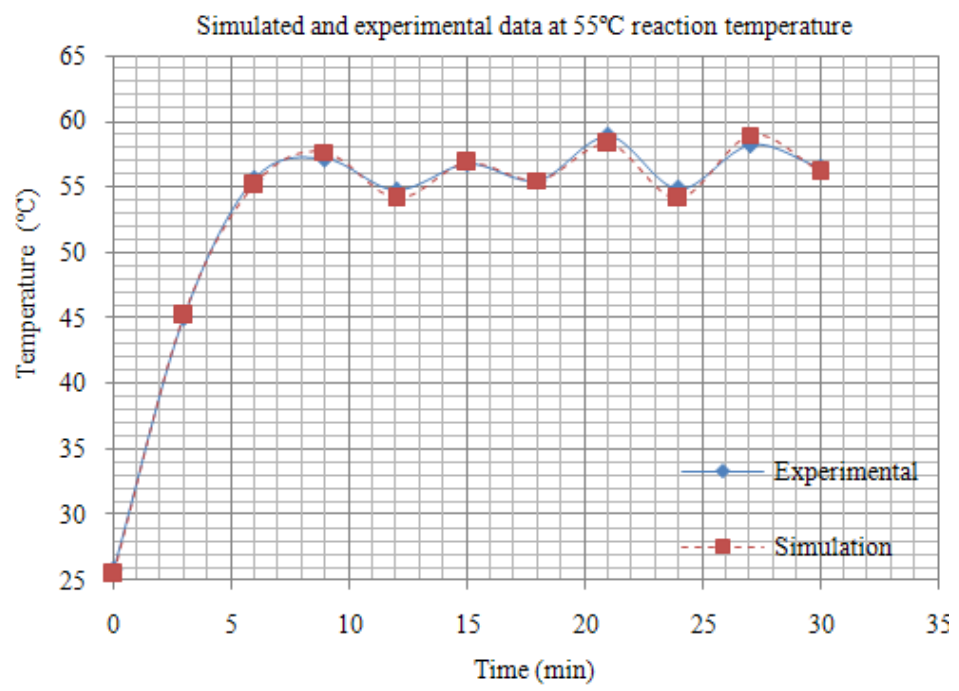

Fig. 9: Simulated temperature (from power absorbed function) and experimental temperature at $55^{\circ} \mathrm{C}$

Also, it must be pointed out that the method of using IR thermometer does not yield the core temperature of the reaction mixture as it only detects the temperature of the surface (in this case, the surface of the borosilicate bottle). Considering the complexity of the coupled electromagnetic and thermal physics model and that it does not include moisture migration, a fairly good match is obtained between the measured and simulated values.

Comparison of Efficiency between Experimental and Simulation Results: By obtaining the data from the simulation results, the efficiency of the epoxidation reaction can be calculated. It should be emphasized that discussion about energy efficiency should always relate to whether microwave energy prompts activation of chemical reactions more efficiently. This is a factor that describes the efficiency of the microwave input and must be included in investigations or discussions. Although such discussions had always been neglected due to the fact that most of the reactions are carried out on a millimolar scale, but when it comes a time to consider a scale-up $(\mathrm{mM}>\mathrm{M}$ $>\mathrm{kM}$ ), the limitation of this approach becomes obvious. The determination of the energy input and the energy that is required to reach a certain temperature follows simple physical laws Eq. 14-16:

$$
\begin{aligned}
& \mathrm{Q}_{\mathrm{mw}}=\mathrm{P}_{\mathrm{mw}} \mathrm{t} \\
& \mathrm{Q}_{\mathrm{th}}=\mathrm{mc}_{\mathrm{p}} \Delta \mathrm{T} \\
& \eta=\frac{\mathrm{Q}_{\mathrm{th}}}{\mathrm{Q}_{\mathrm{mw}}}
\end{aligned}
$$

The efficiency factor $\eta$ can be calculated from both the required and the used energy input (Eq. 15). The efficiency factor is dimensionless and describes the effectiveness of the conversion of microwave energy into thermal energy. The available microwave power $\mathrm{P}_{\mathrm{mw}}$ is determined by microwave device manufacturers according to standardized procedures. The values calculated from Eq. (13) seem quite reliable but it should consider the fact that the microwave is pulsed, has a typical conversion of electric energy into microwave energy of 0.5 to 0.65 and has a delay of a few seconds before heating starts. It was found that $7-7.5 \mathrm{~kJ}$ is required to heat the reaction mixture to the reported temperature. The efficiency factor is thus between 0.10 and 0.12 and is far from being an effective conversion of energy.

However, it must be taken into account that the amount reacted is in the milli-Molar (mM) scale and as such gives plenty of room for errors as the sample size is too small. Reliability may be improved by increasing the sample size.

Microwave non-thermal effect: It is clear that there is a certain advantage of power input by microwaves. High power can be applied to reaction mixtures which are able to absorb microwaves in a controlled and fast manner. Microwave irradiation increases the initial reaction rate overall comparing to conventional heating. The rapid reaction was influenced by several factors such as temperature, water content, solvent and substrates. These phenomena can be explained by the following microwave characteristics. One is the particular heat-transfer accomplished by dipole molecular rotation and ion conduction in regular alternating microwave field, which make the heattransfer efficiency higher than that of conventional 
heating. The other is electron spin oscillation of polar molecules caused by the special microwave energy level, which may delicately modulate the local configuration of enzyme molecules to favor efficient binding of substrates according to the well known lock-key theory. The apparent activation energy (Ea) of enzymatic reaction decreases in the microwave field, which is one of the reasons that give an increase in the initial reaction rate.

Under microwave irradiation, the polar molecules (the alcohol and the acid) collide with each other because of thermal effect and microwave effect. "Non-thermal" effects refer to interactions resulting in non-equilibrium energy fluctuation distributions or deterministic, time-averaged drift motion of matter (or both).Therefore, the molecule collision under Microwave Irradiation has extra driving force compared to that under conventional heating, which results in higher rate of reaction under Microwave Irradiation as long as the enzyme is not deactivated by microwave. One of the non-thermal effects is that microwave energy can also modulate the configuration of enzyme molecules by accelerating the molecular rotation and electron spin oscillation of the polar parts of enzyme, which can provide more chance to make the substrates fit to the enzyme in unit of time. The specific nature of this enzyme increases yield tremendously and will prove promising if it is to be used in a large scale.

$\mathrm{Li}$ et al. (2008) the production of zeolite membranes, have speculated on the non-thermal effects of microwave reaction ( $\mathrm{Li}$ and 2008). Apparently, microwave heating could result in different membrane morphology, orientation, composition for the zeolite membranes. And therefore have different permeation characteristics as compared with conventional heating synthesized ones. It was concluded that the function of microwave irradiation during zeolite membrane formation is mainly derived from its thermal effect alongside specific microwave effects, such as "activewater", "selective activation.

Huang et al. (2005) reported that there is microwave effect on substrate specificity of alcohol (Huang et al., 2005). The reaction rate altering can be explained in respect of two important parameterspolarity and steric hindrance effects. The higher the molecular polarity, the stronger the activation resulted from microwave irradiation, in a certain range. It has also been reported that increasing of alcohol carbon numbers makes the polarity effect shrink and the steric hindrance effect dominant. Steric hindrance occurs when the size of groups within a molecule prevents chemical reactions that are observed in related smaller molecules. Although steric hindrance is sometimes a problem, it can also be a very useful tool and is often exploited by chemists to change the reactivity pattern of a molecule by stopping unwanted side-reactions (steric protection).
In this study, the microwave non-thermal effect is apparent as the conventional chemo-enzymatic epoxidation of PAO takes typically 5 hours to complete. In this project, similar to the findings by Huang et al. (2005) an increased reaction rate that is almost 10 times faster has been achieved with just 30 minutes for each reaction. This rate can even be further increased with further adjustments of temperature but has been avoided for fear of deactivating the enzyme.

Along with this finding, it must also be brought to attention that the microwave effect works side by side in the selectivity of the reactant substrates. As it is not conclusive at this point whether one is causal of another or in fact are two different phenomena working together to give increased reaction rates and specificity in substrates chosen for reaction.

Selectivity of microwave method: The effect of microwave irradiation in chemical reactions is a combination of the thermal effect and non-thermal effects, i.e., overheating, hot spots and selective heating and non-thermal effects of the highly polarizing field, in addition to effects on the mobility and diffusion that may increase the probabilities of effective contacts. Acceleration or changes in reactivity and selectivity could be explained by a specific radiation effect and not merely by a thermal effect. However, in some enzymatic reactions, in which reaction rate, yield and selectivity such as stereo-selectivity or region-selectivity are obviously influenced (Lin and Lin, 1998; Parker et al., 1996; Ipsita and Gupta, 2003; Fang et al., 2003; Vacek et al., 2000; Yadav and Lathi, 2004).

In terms of substrate selectivity behavior, the electron spin oscillation of the polar $-\mathrm{OH}$ molecules of the free fatty acid branches in the PAO, when exposed to microwave energy, tend to align themselves to the polarity of the microwave. This simple realignment creates higher instances where the - $\mathrm{OH}$ group in a molecule can be juxtaposed to another and forming the hydrogen bond. And as more of the formations occur, the viscosity in the Epoxidized Palm Acid Oil (EPAO) itself would be increased as the bonds at the molecular level provides resistance to flow. Similar results had been reported by Biswas et al. (2007) wherein dimerization of triacylglycerol molecules as a result of heat treating or microwave irradiation of soybean oil may lead to a decrease in effective polar groups because of the configuration of the dimerized molecule (Brosseau and Beroual, 2003). Suggesting that the decreasing of effective polar groups $(-\mathrm{OH})$ had been due to the formation of new hydrogen bonds. Although it was also reported by Biswas et al. (2007) that microwave irradiation shows promise to improve the cold-flow 
behavior of soybean oil, the treatment apparently will not produce superior vegetable oil-based lubricants (Sahoo and Biswas, 2009). As such the EPAO produced with this method would have to be blended with stock lubricants and tested for increased lubricity without much decline in resilience to the mechanical shear stress and heat that it will be subjected to in everyday usage.

In the most general sense, the reaction mixture in this study can be considered a composite material as it is the combination of solvent, trygleceride, free fatty acids, peracid and enzyme. They are present as separated components with different chemical and dielectric properties. It is understood from Brosseau's work that the presence of a small concentration of conductive filler such as carbon black particles in a thermosetting polymer host can affect dramatically the dielectric properties of the composite material (Fang et al., 2003). At low filler concentrations, the samples reflect the properties of the matrix material. At a concentration threshold, there is a crossover between an insulating behavior (in which the carbon black particles behave as isolated aggregates) and a conductor behavior corresponding to the formation of infinitely large connected clusters of carbon black aggregates, creating a connection between the electrodes, i.e. the so called percolation paths or channels. Similarly, in the epoxidation reaction, every single component gives the reaction an attributive outcome. There is a complication to the development of a detailed dielectric understanding for this system. There is a need for models that incorporate basic properties of individual constituents and predict the actual properties of the composites before any experimental investigations need to be performed.

\section{CONCLUSION}

In this study, a quantitative view of a microwave assisted process of enzymatic epoxidation has been established. The development and experimental validation of a comprehensive $3 \mathrm{D}$ electromagnetic and heat transfer model of the epoxidation process has been achieved. Three-dimensional partial differential equations are formulated for the electromagnetic field and heat and mass transfer processes. The electromagnetic simulation results of the power absorbed and temperature distribution of the reaction process can be related to real-time experimentation results and thus are used to predict the energy of the epoxidation reaction. The energy used by the microwave irradiated reaction is by far much lesser than the energy used in conventional heating. However, in terms of the energy efficiency for the power absorbed by the reactant and the power consumed by the microwave oven, it had fared badly with only a $10-12 \%$ efficiency.

The research undertaken has:
- Improved the epoxidation process of Palm Acid Oil by incorporating enzyme as a catalyst and using microwave heating. It has been shown that epoxidation can be done rapidly and at a lower level of activation energy of $27.767 \mathrm{~kJ} \mathrm{~mol}^{-1}$. It is a green process with increased efficiency as it reduced a typically 5-hour reaction to less than an hour

- Successfully developed and experimentally validated a 3D electromagnetic heat transfer model. The electromagnetic simulation results of the power absorbed and temperature distribution concurs with real-time experimentation temperature results. This model can thus be used to predict the energy of the epoxidation and other future microwave reactions

- Shown the existence of Microwave Non-Thermal Effects and Microwave Selectivity in Substrate Specificity. The increased reaction rate from typical chemo-enzymatic epoxidation reactions can be explained by dipole molecular rotation, ion conduction in electron spin oscillation and the lock-key theory of how enzymes tend to align the substrates ready for reactions, especially under microwave exposure

Among the aspects that have not been given consideration in this study and whose importance should be evaluated by the development of more advanced models are the simulation of the chemical energy involved in the reaction (achievable by CHEMKIN ${ }^{\mathrm{TM}}$ ), inclusion of the effects of stirring on heat redistribution and microwave absorption by some of the volatile products. Also, a secondary reaction mechanism should be considered to obtain sound predictions of the product yields. A further analysis about the influences of the medium's dielectric properties and the size/shape of both sample and microwave cavity are also needed for the successive steps of technology design and optimization. Detailed measurements of the field variables and yields and composition of epoxidation products are also needed for improving the knowledge of the process fundamentals and for experimental validation of comprehensive models.

More in-depth studies should be conducted in these areas as the development of Palm Acid Oil or any derivatives of Palm Oil into the production of renewable lubricants is a worthy venture, in sync with our current market and environmental needs. The rapid and green method presented here could well be fine-tuned and eventually scaled-up to produce a lubricant that is comparable to the products that are currently in use.

\section{REFERENCES}

Albi, T., A. Lanz'on, A. Guinda, M.C. P'erezCamino and M. Le'on, 1997. Microwave and conventional heating effects on some physical and chemical parameters of edible fats. J. Agric. Food Chem., 45: 3000-3003. DOI: $10.1021 /$ jf970168c 
Biswas, A., A. Adhvaryu, D.G. Stevenson, B.K. Sharma and J.L. Willet et al., 2007. Microwave irradiation effects on the structure, viscosity, thermal properties and lubricity of soybean oil. Indust. Crops Prod., 25: 1-7. DOI: 10.1016/j.indcrop.2006.04.001

Brosseau, C. and A. Beroual, 2003. Computational electromagnetics and the rational design of new dielectric heterostructures. Progress Mat. Sci., 48: 373-456. DOI: $10.1016 /$ S00796425(02)00013-0

Caddick, S. and R. Fitzmaurice, 2009. Microwave enhanced synthesis. Tetrahedron, 65: 3325-3355. DOI: 10.1016/j.tet.2009.01.105

Fang, Y., W. Huang and Y.M. Xia, 2003. Consecutive microwave irradiation induced substrate inhibition on the enzymatic esterification. Process Biochem., 43: 306-310. DOI: 10.1016/j.procbio.2007.12.006

Honghai, D., Y. Liting, L. Bo, Y. Aihua and S. Guang, 2007. Studies on the kinetics of epoxidation of soybean oil. University GuangZhou. http://www.mdpi.net/cji/cji/2007/09a044pe.htm

Horikoshi, S. and S. Serpone, 2009. Photochemistry with microwaves: Catalysts and environmental applications. J. Photochem. Photobio: Photochem. Rev., 10: 96-110. DOI: 10.1016/j.jphotochemrev.2009.06.001

Huang, W., Y.M. Xia, H. Gao, Y.J. Fang and Y. Wang et al., 2005. Enzymatic esterification between n-alcohol homologs and n-caprylic acid in non-aqueous medium under microwave irradiation. J. Molecular Catalysis B: Enzymatic, 35: 113-116. DOI: 10.1016/j.molcatb.2005.06.004

Hwang, H.S., A. Adhvaryu and S.Z. Erhan, 2003. Preparation and properties of lubricant basestocks from epoxidized soybean oil and 2ethylhexanol. J. Am. Oil Chem. Soc., 80: 811-815. DOI: 10.1007/s11746-003-0777-y

Ipsita, R. and M.N. Gupta, 2003. Non-thermal effects of microwaves on protease-catalyzed esterification and transesterification. Tetrahedron, 59: 5431-5436. DOI: 10.1016/S0040-4020(03)00867-6

Jumbe, A.S. and N. Nandini, 2009. Heavy metals analysis and sediment quality values in urban lakes. Am. J. Environ. Sci., 5: 678-687. DOI: 10.3844/ajessp.2009.678.687

Kappe, C.O., 2004. Controlled microwave heating in modern organic synthesis. Angew Chem. Int. Ed. Engl., 43: 6250-6284.

Kuntom, A., W.L. Siew and Y.A. Tan, 1994. Characterization of Palm Acid Oil. JAOCS, 71: 525-528. DOI: $10.1007 / \mathrm{BF} 02540665$
Li, Y. and W. Yang, 2008. Microwave synthesis of zeolite membranes: A review. J. Membrane Sci., 316: 3-17. DOI: 10.1016/j.memsci.2007.08.054

Lin, G.L. and W.Y. Lin, 1998. Microwavepromoted lipase-catalyzed reactions. Resolution of ( \pm )-1-Phenylethanol. J. Org. Chem., 61: 77467749. DOI: $10.1021 /$ jo960309u

MacKenzie, K., O. Dunens and A. Harris, 2009. A review of carbon nanotube purification by microwave assisted acid digestion. Sep. Purif. Technol., 66: 209-222. DOI: 10.1016/j.seppur.2009.01.017

Orellana-Coca, C., Tornvall U., Adlercreutz D., Hatti-Kaul R., 2005. Chemo-enzymatic epoxidation of oleic acid and methyl oleate in solvent-free medium. Biocatal Biotransform, 23:431-437. DOI: 10.1080/10242420500389488

Parker, M.C., T. Besson, S. Lamare and M.D. Legoy, 1996. Microwave radiation can increase the rate of enzyme-catalysed reactions in organic media. Tetrahedron Lett., 37: 8383-8386. DOI: 10.1016/0040-4039(96)01544-4

Pillai, U.R., E. Shale-Demessie and R.S. Varma, 2002. Microwave-expedited olefin epoxidation over hydrotalcites using hydrogen peroxide and acetonitrile. Tetrahedron Lett., 43: 2909-2911. DOI: 10.1016/S0040-4039(02)00426-4

Rüsch gen, M.R. and S. Warwel, 1999. Complete and partial epoxidation of plant oils by lipasecatalyzed perhydrolysis. Indust. Crops Prod., 9: 125-132. DOI: 10.1016/S0926-6690(98)00023-5

Sahoo, R.R. and S.K. Biswas, 2009. Frictional response of fatty acids on steel. J. Coll. Interface Sci., 33: 707-718. DOI: 10.1016/j.jcis.2009.01.046

Saifuddin, N. and S A. Fazlili, 2009. Effect of microwave and ultrasonic pretreatments on biogas production from anaerobic digestion of palm oil mill effleunt. Am. J. Eng. Applied Sci., 2: 139-146.

Tan, C.P., Y.B.C. Man, S. Jinap and M.S.A. Yusoff, 2001. Effects of microwave heating on changes in chemical and thermal properties of vegetable oils. J. Am. Oil Chem. Soc., 78: 1227-1232. DOI: $10.1007 / \mathrm{s} 11745-001-0418-5$

Thomas, M.B. and L. Dozier, 2010. Finite element modeling of transient temperatures in a smallcaliber projectile. Am. J. Eng. Applied Sci., 3: 355-362.

Tornvall,U., C. Orellana-Coca, R. Hatti-Kaul and D. Adlercreutz, 2007. Stability of immobilized Candida antarctica lipase B during chemoenzymatic epoxidation of fatty acids. Enzyme Microbial Technol., 40: 447-451. DOI: 10.1016/j.enzmictec.2006.07.019 
Vacek, M., M. Zarevucka, Z. Wimmer, K. Stransky and K. Demnerova et al., 2000. Selective enzymic esterification of free fatty acids with nbutanol under microwave irradiation and under classical heating. Biotechnol. Lett., 22: 1565-1570. DOI: 10.1023/A:1005637018167
Yadav, G.D. and P.S. Lathi, 2004. Microwave assisted enzyme catalysis for synthesis of n-butyl dipheyl methyl mercapto acetate in non-aqueous media. Clean Techolog. Environ. Policy, 9: 281-287. DOI: $10.1007 / \mathrm{s} 10098-006-0082-3$ 